\title{
Effect of the interaction between the amount and duration of alcohol consumption and tobacco smoking on the risk of esophageal cancer: A case-control study
}

\author{
JUN CHEN $^{1}$, NAN ZHANG ${ }^{1}$, TOSHIFUMI WAKAI ${ }^{2}$, LIZHEN WEI ${ }^{5}$, YUTONG HE ${ }^{5}$, \\ NAOKO KUMAGAI ${ }^{4}, \mathrm{KEIKO} \mathrm{KITSU}^{1}$, SHIJIE WANG ${ }^{5}$ and KOHEI AKAZAWA ${ }^{1,3}$ \\ ${ }^{1}$ Department of Medical Informatics, ${ }^{2}$ Division of Information Science and Biostatistics, \\ Niigata University Graduate School of Medicine and Dental Sciences; ${ }^{3}$ Department of Medical Informatics, \\ Niigata University Medical and Dental Hospital, Niigata; ${ }^{4}$ Biostatistics and Clinical Epidemiology, University of Toyama \\ Graduate School, Toyama, Japan; ${ }^{5}$ The Fourth Affiliated Hospital of Hebei Medical University, Hebei, P.R. China
}

Received July 22, 2010; Accepted September 27, 2010

DOI: $10.3892 /$ etm.2010.152

\begin{abstract}
The effects of alcohol consumption and tobacco smoking on the prevalence of esophageal cancer vary considerably by country, race and lifestyle. Few data exist on the effect of the interaction between the amount and duration of alcohol consumption and tobacco smoking on the incidence of esophageal cancer. In this case-control study, the cases included patients with histologically confirmed esophageal squamous cell carcinoma (ESCC) younger than 60 years of age and recruited between January 1, 2002 and December 31, 2006. The controls had no abnormality during a medical checkup. A total of 835 pairs were created by pairing each case to a gender- and age-matched control. Conditional logistic regression analysis was used to estimate adjusted odds ratios (ORs) and 95\% confidence intervals. Univariate conditional logistic regression analyses revealed that the ORs according to both duration of alcohol consumption and tobacco smoking increased monotonically. Alcohol consumption and tobacco smoking may have a synergistic effect on the incidence of ESCC. Conditional logistic regression analysis using a forward stepwise selection procedure revealed that the incidence of ESCC was associated with the duration of tobacco smoking, the interaction between the amount and duration of alcohol consumption, and a family history of cancer. In particular, groups with a long duration of alcohol consumption and high alcohol intake had much higher ORs than those with short duration and low intake, which highlights the importance of the interaction between the amount and duration of alcohol
\end{abstract}

Correspondence to: Dr Jun Chen, Department of Medical Informatics, Niigata University Graduate School of Medicine and Dental Sciences, Asahimachi-Dori 1-754, Niigata 951-8520, Japan

E-mail: chenjun@med.niigata-u.ac.jp

Key words: alcohol consumption, tobacco smoking, esophageal cancer, interaction between amount and duration intake. This study confirmed the significance of the interaction between alcohol consumption and tobacco smoking in esophageal cancer. This interaction between amount and duration is an accurate indicator for estimating the risk of esophageal cancer attributable to alcohol consumption and tobacco smoking. These findings suggest that decreasing the number of young and middle-aged drinkers and smokers will reduce the incidence of esophageal cancer.

\section{Introduction}

Alcohol consumption and tobacco smoking are established risk factors for esophageal cancer (1-4), and most studies have noted that the dose-response relationship is steep and monotonic. Enzinger and Mayer reported that, in most developed countries, tobacco smoking is associated with a 2- to 4-fold increased risk of esophageal cancer, as compared to non-smokers (5). In addition, Zambon et al reported that the esophageal cancer risk rose steeply with increasing alcohol consumption (6). Furthermore, alcohol and tobacco have been reported to interact multiplicatively in the development of esophageal cancer. Brown et al indicated that the odds ratio (OR) of esophageal squamous cell carcinoma (ESCC), with regard to heavy exposure to both these factors was 35.4 in white males and 149.2 in black males, as compared to non-smokers/light smokers and drinkers of corresponding race (7).

In general, the risk of cancer incidence is estimated in epidemiological studies by using ORs. However, since incidence is affected by the type of alcohol and tobacco consumed, and by race, diet, genetic diversity in susceptibility to alcohol and tobacco, and other socioeconomic factors, there are considerable differences in the ORs of esophageal cancer for the different countries and regions where such research has been conducted (8-14). Consequently, great care must be taken in the interpretation of these estimated ORs. In addition, ORs must be calculated for each country and region (14).

There have been numerous investigations of the associations of the incidence of esophageal cancer with the amount and duration of alcohol consumption and tobacco smoking, 
and with the interaction between and dose-dependency of alcohol consumption and tobacco smoking $(7,10,12,15)$. However, few studies have examined the effects of the interaction between the amount and duration of alcohol consumption and tobacco smoking. Such an examination is of great importance, since the effect on the incidence of esophageal cancer of consuming $40 \mathrm{~g}$ of ethanol per day for 10 years may be quite different from that of consuming $20 \mathrm{~g}$ of ethanol per day for 20 years, even though the total quantity of ethanol consumed is equal.

This study investigated the independent and combined effects of alcohol consumption and tobacco smoking on the risk of esophageal cancer in Hebei Province, China. In particular, we examined the interaction between the amount and duration of alcohol consumption and tobacco smoking.

\section{Patients and methods}

Study design. A matched-pair case-control study was conducted to estimate the individual and combined risks of alcohol consumption and tobacco smoking. The subjects included 835 pairs, each of which consisted of a patient with esophageal cancer (case) and a healthy individual (control) matched by age ( \pm 2 years) and gender. Eligible subjects had been residents of the city of Shijiazhuang, Hebei Province, China, for at least 20 years and were younger than 60 years of age. Before being interviewed, the subjects were informed of all study objectives and the content of the research. The research protocol was approved by the Local Ethics Committee and the Research Committee of Hebei Medical University.

Subjects. The cases were drawn from 1,911 patients who had received a diagnosis of primary invasive cancer of the esophagus between January 2002 and December 2006 at the Fourth Hospital of Hebei Medical University in Shijiazhuang. All diagnoses were histologically confirmed and based on the International Classification of Diseases, 10th revision (ICD-10, code C15). From these 1,911 patients, we excluded 235 who had suffered a recurrence of esophageal cancer or had another concomitant cancer, as well as 720 patients who were older than 60 years. Thus, 956 cases remained for inclusion. The pathological diagnosis was squamous cell carcinoma in 835 cases $(87.3 \%)$, adenocarcinoma in 35 cases $(3.7 \%)$ and other cancer types in 86 cases $(9 \%)$. The 835 cases with squamous cell carcinoma - the dominant histological type - were enrolled for case-control matching and subsequent statistical analysis.

Using a physical examination database, 3,076 individuals who lived in the same area (Hebei Province, China) from 2004 to 2006 were enrolled as controls and underwent medical checkups.

Risk factors. The factors and categories used in the study are shown in Table I. Data for the study were obtained from the subjects' medical records and, in the case of controls, from checkups. The information collected included age at diagnosis (for all cases), age at medical checkup (for all controls), gender, alcohol and tobacco use, medical history and family history of cancer. Participants were interviewed to collect details on average daily tobacco use, ages when tobacco smoking began and ended, and duration of smoking.

Information on alcohol consumption consisted of the average grams of ethanol consumed per day, type of alcoholic beverage consumed, lifetime duration of the habit, ages when alcohol consumption began and ended, and number of years since quitting. The type of beverage consumed was classified as baijiu, beer and other liquors. Baijiu is a Chinese distilled alcoholic beverage usually made from sorghum or glutinous rice. It is the most popular drink in China and contains $40-60 \%$ ethanol per volume on average. Chinese usually drink baijiu in a single swallow from a 20 - to $30-\mathrm{ml}$ glass, without a chaser. The average daily amount of alcohol was calculated using ethanol exchange rates of $40 \%$ for baijiu, $5 \%$ for beer, $12 \%$ for wine and $40 \%$ for hard liquor. A non-drinker was defined as someone who consumed less than one drink per month. One drink was defined as $50 \mathrm{ml}$ of baijiu, $400 \mathrm{ml}$ of beer or $50 \mathrm{ml}$ of hard liquor. The total amount of ethanol consumed per day was calculated using the above-mentioned ethanol percentages. The resulting values were converted to grams by multiplying $1 \mathrm{ml}$ of pure ethanol by 0.789 (16).

Finally, the risk factors used in this study were summarized with respect to the amount of alcohol consumed per day, duration of alcohol consumption (years), interaction between the amount and duration of alcohol consumption (the total amount of alcohol consumed over a certain time period), number of cigarettes smoked per day, duration of tobacco smoking (years), interaction between the amount and duration of tobacco smoking (the total amount of tobacco smoked over a certain time period) and family history of cancer. The interaction between the amount and duration of alcohol consumption for $20 \mathrm{~g}$ of alcohol per day for 10 years and that for $10 \mathrm{~g}$ of alcohol per day for 20 years were distinguished by slightly modifying partial dummy variables, as described in the next section.

Statistical analysis. We performed conditional logistic regression analysis to estimate ORs and corresponding 95\% confidence intervals (CIs). A forward stepwise regression method was used to select the best subset of risk factors associated with the incidence of esophageal cancer, after adjusting for the effect of other factors. The candidate factors were the amount of alcohol consumed, duration of alcohol consumption, interaction between the amount and duration of alcohol consumption, amount of tobacco smoked, duration of tobacco smoking, interaction between the amount and duration of tobacco smoking, and family history of cancer. The amount and duration were divided into four categories. These categories were then converted into three categories by using partial dummy variables; the reference category included those individuals who were non-smokers and/or non-drinkers. For the interaction between the amount and duration of tobacco smoking and the interaction between the amount and duration of alcohol consumption, dummy variables were created so that individuals smoking 10 cigarettes per day for 20 years could be discriminated from those smoking 20 cigarettes per day for 10 years, even though the product for both is 200. All statistical analyses were performed using SAS 9.1. A p-value $<0.05$ was considered statistically significant for all tests. 
Table I. Characteristics of the cases and controls.

\begin{tabular}{|c|c|c|c|}
\hline \multirow[b]{2}{*}{ Factors/categories } & \multicolumn{3}{|c|}{ No. of subjects (\%) } \\
\hline & Cases & Controls & Total \\
\hline \multicolumn{4}{|l|}{ Age (years) ${ }^{a}$} \\
\hline$<45$ & $78 \quad(9.3)$ & 78 (9.3) & $156(9.3)$ \\
\hline 45 to $<60$ & $757(90.7)$ & $757(90.7)$ & $1,514(90.7)$ \\
\hline \multicolumn{4}{|l|}{ Gender } \\
\hline Male & $571(68.4)$ & $571(68.4)$ & $1,142(68.4)$ \\
\hline Female & $264(31.6)$ & $264(31.6)$ & $528(31.6)$ \\
\hline \multicolumn{4}{|c|}{ Family history of cancer } \\
\hline Yes & $230(27.5)$ & $122(14.6)$ & $352(21.1)$ \\
\hline No & $605(72.5)$ & $713(85.4)$ & $1,318(78.9)$ \\
\hline \multicolumn{4}{|c|}{ Duration of alcohol consumption (years) } \\
\hline 0 & $530(63.5)$ & $613(73.4)$ & $1,143(68.4)$ \\
\hline$<15$ & $62(7.4)$ & $59(7.1)$ & $121(7.2)$ \\
\hline 15 to $<20$ & $133(15.9)$ & $122(14.6)$ & $255(15.3)$ \\
\hline$\geq 20$ & $110(13.2)$ & 41 (4.9) & $151(9.0)$ \\
\hline \multicolumn{4}{|c|}{ Amount of alcohol intake (g/day) } \\
\hline 0 & $530(63.5)$ & $613(73.4)$ & $1,143(68.4)$ \\
\hline$<30$ & $78 \quad(9.3)$ & 40 (4.8) & $118(7.0)$ \\
\hline 30 to $<60$ & $99(11.9)$ & $107(12.8)$ & $206(12.3)$ \\
\hline$\geq 60$ & $128(15.3)$ & $75(9.0)$ & $203(12.2)$ \\
\hline \multicolumn{4}{|c|}{ Duration of tobacco smoking (years) } \\
\hline 0 & $427(51.1)$ & $483(57.8)$ & $910(54.5)$ \\
\hline$<20$ & $56(6.7)$ & $82(9.8)$ & $138 \quad(8.3)$ \\
\hline 20 to $<30$ & $160(19.2)$ & $140(16.8)$ & $300(18.0)$ \\
\hline$\geq 30$ & $192(23.0)$ & $130(15.6)$ & $322(19.3)$ \\
\hline \multicolumn{4}{|c|}{ Amount of tobacco smoking (cigarettes/day) } \\
\hline 0 & $427(51.1)$ & $483(57.8)$ & $910(54.5)$ \\
\hline$<10$ & $102(12.2)$ & $69 \quad(8.3)$ & $171(10.2)$ \\
\hline 10 to $<20$ & 205 (24.6) & 203 (24.3) & $408(24.4)$ \\
\hline$\geq 20$ & $101(12.1)$ & $80 \quad(9.6)$ & $181(10.8)$ \\
\hline
\end{tabular}

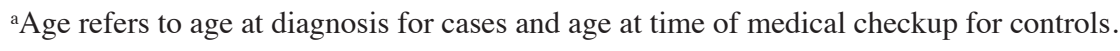

\section{Results}

Subject characteristics. Table I shows the distribution of cases and controls with respect to age, gender and risk factors. There were 757 pairs comprised of participants over the age of 45 years. The proportion of males was $68.4 \%$. Table II shows the distribution of cases and controls for each risk factor. The distribution of exposure to alcohol consumption, tobacco smoking and family history of cancer was asymmetric between cases and controls, i.e., the levels of alcohol consumption and tobacco smoking were higher among cases than among controls. For example, as shown in Table IIB, there were 49 pairs in which the cases consumed 0 to $<30 \mathrm{~g}$ of ethanol and the controls consumed 0 units; however, there were only 8 pairs in which the cases consumed 0 units and the controls consumed 0 to $<30$ units. Similarly, the duration of alcohol consumption and tobacco smoking was longer for the cases than for the controls.
Estimation of the risk of alcohol consumption and tobacco smoking by univariate analysis. Conditional logistic regression analysis was performed to estimate the risk of the incidence of esophageal cancer. Table III shows the ORs and corresponding $95 \%$ CIs obtained from the univariate conditional logistic regression analysis. With respect to the reference category, the ORs of esophageal cancer increased monotonically with the duration of alcohol consumption and tobacco smoking. Regarding the duration of alcohol consumption, the ORs of esophageal cancer for the categories of 0-15, 15-20 and $>20$ years were $1.22,1.26$ and 3.10, respectively. The OR of esophageal cancer for alcohol consumption $>20$ years differed significantly from that for the reference (no consumption of alcohol) category $(\mathrm{p}<0.001)$. Regarding tobacco smoking, the ORs of esophageal cancer for the categories of 0-20, 20-30 and $>30$ years were $0.77,1.29$ and 1.67 , respectively; the OR for a duration of smoking $>30$ years was significantly higher than that for non-smokers $(\mathrm{p}<0.001)$. However, the associations 
Table II. Amount and duration of alcohol and tobacco consumption in the matched case-control pairs.

A, Duration of alcohol consumption (years).

\begin{tabular}{lrrcrr}
\hline Controls & \multicolumn{5}{c}{ Cases } \\
\cline { 2 - 6 } & 0 & $<15$ & 15 to $<20$ & $\geq 20$ & Total \\
\cline { 2 - 6 } & 515 & 32 & 44 & 22 & 613 \\
0 & 9 & 10 & 25 & 15 & 59 \\
$<15$ & 6 & 14 & 48 & 54 & 122 \\
15 to $<20$ & 0 & 6 & 16 & 19 & 41 \\
$\geq 20$ & 530 & 62 & 133 & 110 & 835 \\
Total & & & & & \\
\hline
\end{tabular}

B, Amount of alcohol consumption (g/day).

\begin{tabular}{lrrcrr}
\hline Controls & \multicolumn{5}{c}{ Cases } \\
\cline { 2 - 6 } & 0 & $<30$ & 30 to $<60$ & $\geq 60$ & Total \\
\cline { 2 - 6 } & 515 & 49 & 35 & 14 & 613 \\
0 & 8 & 11 & 17 & 4 & 40 \\
$<30$ & 5 & 17 & 43 & 42 & 107 \\
30 to $<60$ & 2 & 1 & 4 & 68 & 75 \\
$\geq 60$ & 530 & 78 & 99 & 128 & 835 \\
Total & & & & & \\
\hline
\end{tabular}

C, Duration of tobacco smoking (years).

\begin{tabular}{lrrcrr}
\hline Controls & \multicolumn{5}{c}{ Cases } \\
\cline { 2 - 6 } & 0 & $<20$ & 20 to $<30$ & $\geq 30$ & Total \\
\cline { 2 - 6 } & 384 & 21 & 41 & 37 & 483 \\
0 & 13 & 11 & 29 & 29 & 82 \\
$<20$ & 6 & 18 & 58 & 58 & 140 \\
20 to $<30$ & 24 & 6 & 2 & 68 & 130 \\
$\geq 30$ & 427 & 56 & 160 & 192 & 835 \\
Total & & & & & \\
\hline
\end{tabular}

D, Amount of tobacco smoking (cigarettes/day).

\begin{tabular}{lrrcrr}
\hline Controls & \multicolumn{5}{c}{ Cases } \\
\cline { 2 - 6 } & 0 & $<10$ & 10 to $<20$ & $\geq 20$ & Total \\
\cline { 2 - 6 } & 384 & 41 & 36 & 22 & 483 \\
0 & 6 & 16 & 40 & 7 & 69 \\
$<10$ & 26 & 35 & 95 & 47 & 203 \\
10 to $<20$ & 11 & 10 & 34 & 25 & 80 \\
$\geq 20$ & 427 & 102 & 205 & 101 & 835 \\
Total & & & & &
\end{tabular}

E, Family history of cancer.

\begin{tabular}{lrrr}
\hline Controls & \multicolumn{3}{c}{ Cases } \\
\cline { 2 - 4 } & No & Yes & Total \\
\hline No & 519 & 194 & 713 \\
Yes & 86 & 36 & 122 \\
Total & 605 & 230 & 835 \\
\hline
\end{tabular}

of the OR for esophageal cancer with the amount of alcohol consumed and with the amount of tobacco smoked were not monotonic.

Interaction between alcohol consumption and tobacco smoking. Table IV illustrates the results of conditional logistic regression analysis of the interaction between alcohol consumption and tobacco smoking. Table IV-A shows the distribution of alcohol consumption and tobacco smoking in the matched pairs. Table IV-B shows the regression coefficients and standard errors of the four possible states, i.e., neither drinking nor smoking, drinking only, smoking only, and both drinking and smoking. Table IV-C shows the estimated ORs of esophageal cancer according to alcohol consumption, tobacco smoking and interaction between the two factors, which were 19.5, 6.2 and 43.8 , respectively.

Effect of the interaction between the amount and duration of alcohol consumption and tobacco smoking. Table $\mathrm{V}$ shows the ORs for esophageal cancer according to different combinations of alcohol and tobacco amounts and duration. Multiple conditional logistic regression analysis was performed in a forward stepwise manner, and the following seven factors were used as candidates: the amount and duration of tobacco smoking, amount and duration of alcohol consumption, interaction between the amount and duration of tobacco smoking, interaction between the amount and duration of alcohol consumption, and family history of cancer.

Dduration of tobacco smoking, interaction between the amount and duration of alcohol consumption, and family history of cancer were selected as the independent and significant variables. However, the interaction between the amount and duration of tobacco smoking and the amount and duration of alcohol were not selected in this stepwise regression analysis. We found that the OR of the group with the shortest duration and lowest amount of alcohol consumption was 6.99, and that the lowest OR for esophageal cancer among drinkers was 5.23. Individuals who consumed $\geq 60 \mathrm{~g}$ alcohol per day had an OR $>40$, regardless of the duration of alcohol consumption. Indeed, those consuming $\geq 60 \mathrm{~g}$ alcohol per day for longer than 20 years had an estimated OR of 183.1.

\section{Discussion}

Epidemiological studies have shown that the combination of moderate/heavy consumption of alcohol, tobacco smoking, infrequent consumption of raw fruits and vegetables, and low annual income accounts for almost all cases of esophageal cancer (17-21). Among these factors, alcohol consumption and tobacco smoking are the predominant risk factors for this cancer, i.e., the disease is more frequent among smokers and alcohol drinkers. Brown et al (24) reported that the populationattributable risk of esophageal cancer due to the combination of alcohol consumption and tobacco smoking was higher than $90 \%$ in both whites and blacks in the US $(10,19)$. Studies in other countries have reported similar findings $(12,22)$. However, the ORs for the effects of alcohol and smoking on the incidence of esophageal cancer have varied considerably in well-designed epidemiological studies. Therefore, it would appear desirable to calculate estimates of the risk of esopha- 
Table III. Odds ratios (ORs) and 95\% confidence intervals (CIs) for each risk factor upon univariate analysis.

\begin{tabular}{|c|c|c|c|}
\hline Factors & OR & $95 \% \mathrm{CI}$ & P-value \\
\hline \multicolumn{4}{|c|}{ Duration of alcohol consumption (years) } \\
\hline 0 & 1.00 & & \\
\hline$<15$ & 1.22 & $0.84-1.77$ & 0.310 \\
\hline 15 to $<20$ & 1.26 & $0.96-1.66$ & 0.090 \\
\hline$\geq 20$ & 3.10 & $2.13-4.52$ & $<0.001$ \\
\hline \multicolumn{4}{|c|}{ Amount of alcohol consumption (g/day) } \\
\hline 0 & 1.00 & & \\
\hline$<30$ & 2.26 & $1.51-3.35$ & $<0.001$ \\
\hline 30 to $<60$ & 1.07 & $0.80-1.44$ & 0.650 \\
\hline$\geq 60$ & 1.97 & $1.45-2.69$ & $<0.001$ \\
\hline \multicolumn{4}{|c|}{ Duration of tobacco smoking (years) } \\
\hline 0 & 1.00 & & \\
\hline$<20$ & 0.77 & $0.54-1.11$ & 0.160 \\
\hline 20 to $<30$ & 1.29 & $1.00-1.68$ & 0.050 \\
\hline$\geq 30$ & 1.67 & $1.29-2.16$ & $<0.001$ \\
\hline \multicolumn{4}{|c|}{ Amount of tobacco smoking (cigarettes/day) } \\
\hline 0 & 1.00 & & \\
\hline$<10$ & 1.67 & $1.20-2.33$ & 0.002 \\
\hline 10 to $<20$ & 1.14 & $0.90-1.44$ & 0.260 \\
\hline$\geq 20$ & 1.42 & $1.04-1.97$ & 0.030 \\
\hline
\end{tabular}

Table IV. Conditional logistic regression analysis of the interaction between alcohol consumption and tobacco smoking.

A, Distribution of alcohol consumption and tobacco smoking in matched pairs of cases and controls.

\begin{tabular}{|c|c|c|c|c|c|}
\hline \multirow[t]{2}{*}{ Controls } & \multicolumn{5}{|c|}{ Cases } \\
\hline & Neither & Alcohol consumption only & Tobacco smoking only & Both & Total \\
\hline Neither & 365 & 11 & 50 & 3 & 429 \\
\hline Alcohol consumption only & 3 & 5 & 3 & 43 & 54 \\
\hline Tobacco smoking only & 2 & 19 & 98 & 65 & 184 \\
\hline Both & 3 & 19 & 6 & 140 & 168 \\
\hline Total & 373 & 54 & 157 & 251 & 835 \\
\hline
\end{tabular}

$\mathrm{B}$, Results of conditional logistic regression analysis.

\begin{tabular}{lcc}
\hline Variables & Regression coefficient & Standard error \\
\hline Neither & 1.00 & - \\
Alcohol consumption only & 2.97 & 0.48 \\
Tobacco smoking only & 1.83 & 0.38 \\
Both & -1.01 & 0.45 \\
\hline
\end{tabular}

\section{C, Estimated ORs.}

Alcohol consumption

Tobacco smoking

\begin{tabular}{lrr}
\cline { 2 - 3 } & No & Yes \\
\hline No & 1.0 & 6.2 \\
Yes & 19.5 & 43.8 \\
\hline
\end{tabular}


Table V. The odds ratios (ORs) for esophageal cancer, by duration and dose intensity of exposure, as determined by conditional logistic regression analysis.

\begin{tabular}{|c|c|c|c|c|c|}
\hline Factors & Cases & Controls & OR & $95 \% \mathrm{CI}$ & P-value \\
\hline \multicolumn{6}{|l|}{ Duration of tobacco smoking (years) } \\
\hline 0 & 427 & 483 & - & - & - \\
\hline$<20$ & 56 & 82 & 1.71 & $0.98-2.95$ & 0.060 \\
\hline 20 to $<30$ & 160 & 140 & 3.68 & $2.23-6.08$ & $<0.001$ \\
\hline$\geq 30$ & 192 & 130 & 4.76 & $2.86-7.94$ & $<0.001$ \\
\hline \multicolumn{6}{|c|}{ Dose intensity of alcohol consumption } \\
\hline 0 & 530 & 613 & - & - & - \\
\hline$<15$ years and $<30 \mathrm{~g}$ & 33 & 20 & 6.99 & $3.14-15.56$ & $<0.001$ \\
\hline 15 to $<20$ years and $<30 \mathrm{~g}$ & 27 & 16 & 8.86 & $3.53-22.26$ & 0.002 \\
\hline$\geq 20$ years and $<30 \mathrm{~g}$ & 18 & 4 & 21.56 & $5.28-88.06$ & $<0.001$ \\
\hline$<15$ years and 30 to $<60 \mathrm{~g}$ & 19 & 27 & 5.23 & $2.19-12.46$ & $<0.001$ \\
\hline 15 to $<20$ years and 30 to $<60 \mathrm{~g}$ & 43 & 56 & 9.34 & $4.13-21.11$ & $<0.001$ \\
\hline$\geq 20$ years and 30 to $<60 \mathrm{~g}$ & 37 & 24 & 13.59 & $5.34-34.63$ & $<0.001$ \\
\hline$<15$ years and $\geq 60 \mathrm{~g}$ & 10 & 12 & 45.26 & 10.53-194.56 & $<0.001$ \\
\hline 15 to $<20$ years and $\geq 60 \mathrm{~g}$ & 63 & 50 & 49.94 & $16.65-149.83$ & $<0.001$ \\
\hline$\geq 20$ years and $\geq 60 \mathrm{~g}$ & 55 & 13 & 183.12 & $49.10-682.91$ & $<0.001$ \\
\hline Family history of cancer & & & 2.57 & $1.92-3.43$ & $<0.001$ \\
\hline Yes & 230 & 122 & & & \\
\hline No & 605 & 713 & & & \\
\hline
\end{tabular}

geal cancer for each country, region and race. The present study quantitatively estimated the effects of alcohol consumption and tobacco smoking on the development of esophageal cancer in Chinese adults who were under the age of 60 years and living in the urban area of Hebei Province. Accompanying the increase in age, physiological function and immunosurveillance of the body appear to decrease in the elderly. There are many factors associated with the differentiation and development of cancer in the body of elderly individuals. In this study, we aimed to clarify the contribution of smoking and alcohol consumption to the development of esophageal cancer. To avoid the interference of numerous factors, we selected individuals below 60 years of age as subjects, as suggested by the World Health Organization, who define an elderly population as one over 60 years of age. Based on this, we focused on the interactive effect of alcohol consumption and tobacco smoking, and the interaction between the amount and duration of drinking and smoking.

The interaction between alcohol consumption and tobacco smoking has been extensively studied, and the results indicate that alcohol and tobacco interact in a multiplicative way $(7,19,23)$. In one large-scale study, Castellsagué et al reported that the risk of esophageal cancer among subjects with the highest level of alcohol consumption and highest level of cigarette smoking was increased by 51 - and 35 -fold in men and women, respectively (11). The present case-control study found that the OR of the interaction between alcohol consumption and tobacco smoking was 43.8 , and that alcohol and tobacco interacted in a multiplicative manner, as shown in Table IV. Furthermore, Brown et al estimated the joint ORs for alcohol consumption and tobacco smoking stratified by annual income, using a reference group comprising light smokers and light drinkers (24); the ORs of the interaction ranged from 2.0 to 420.6. Thus, our estimated ORs of the interaction between alcohol and tobacco are in accord with those reported in other reliable studies.

Using conditional logistic regression analysis with a forward stepwise method, we identified the interaction between the amount and duration of alcohol consumption, duration of tobacco smoking and family history of cancer as the subset of significant risk factors that contribute to the incidence of esophageal cancer. Our most salient finding is that the interaction between the amount and duration of alcohol consumption (i.e., the average daily alcohol intake multiplied by the duration of intake) is included in this subset of significant and independent risk factors. This suggests that the interaction between the amount and duration of alcohol consumption has more predictive power than when these two factors are considered separately. By contrast, the interaction between the amount and duration of tobacco smoking had weaker predictive power for the incidence of esophageal cancer than the duration of tobacco smoking. As shown in Table V, heavy drinkers had the highest risk for esophageal cancer, particularly those who had been drinking for many years. Almost all alcohol drinkers in China consume baijiu without mixing it with water. Chronic consumption of beverages with a high ethanol content has been implicated in the development and progression of esophageal cancer.

The strength of our study was the use of a case-control design and the enrollment of sufficient numbers of cases and controls in matched pairs to estimate the effects of the interaction between the amount and duration of alcohol consumption and tobacco smoking and the interaction between alcohol and tobacco. However, this population-based case-control study did have some limitations. We estimated the risks for esophageal cancer using information on only alcohol, smoking and 
family history of cancer, and did not collect data on other possible risk factors, for example, social status and diet. We were unable to obtain precise data on income and diet in healthy individuals in Hebei Province, particularly among agricultural workers. In addition to alcohol and tobacco, dietary factors may play a causal role in the carcinogenesis of esophageal cancer. Unfortunately, the lack of dietary information made it impossible to evaluate such factors. Finally, due to the very small number of individuals who successfully relinquish alcohol drinking or tobacco smoking, we could not include cessation of alcohol drinking or tobacco smoking as a factor in esophageal cancer.

In conclusion, lifestyle modifications, particularly a decreased intake of alcoholic beverages and tobacco smoking, would markedly lower the incidence of ESCC. From a public health standpoint, our study suggests that the greatest impact would come from decreasing consumption of alcoholic beverages, particularly among the $12.2 \%$ of the population who consume more than $60 \mathrm{~g}$ of alcohol per day. We expect that further studies on socioeconomic variables and diet will help to identify a new set of esophageal cancer risk factors that may be amenable to intervention.

\section{References}

1. Nyrén $\mathrm{O}$ and Adami H: Esophageal cancer. In: Textbook of Cancer Epidemiology. Adami HO and Hunter D (eds). Oxford University, Oxford, pp137-161, 2002.

2. Tobacco Smoking. IARC Monogr Eval Carcinog Risk Chem Hum 38: 35-394, 1986.

3. Alcohol Drinking. IARC Working Group, Lyon, 13-20 October, 1987. IARC Monogr Eval Carcinog Risks Hum 44: 1-378, 1988.

4. IARC Working Group on the evaluation of carcinogenic risks to humans: IARC Monographs on the Evaluation of Carcinogenic Risks to Humans Alcohol Drinking. Vol. 44. International Agency for Research on Cancer, Lyon, France, 1988.

5. Enzinger PC and Mayer RJ: Esophageal cancer. N Engl J Med 23: 2241-2252, 2003.

6. Zambon P, Talamini R, La Vecchia C, Dal Maso L, Negri E, Tognazzo S, Simonato L and Franceschi S: Smoking, type of alcoholic beverage and squamous-cell oesophageal cancer in northern Italy. Int J Cancer 86: 144-149, 2000.

7. Brown LM, Hoover RN, Greenberg RS, Schoenberg JB, Schwartz AG, Swanson GM, Liff JM, Silverman DT, Hayes RB and Pottern LM: Are racial differences in squamous cell esophageal cancer explained by alcohol and tobacco use? J Natl Cancer Inst 86: 1340-1345, 1994

8. Castelletto R, Castellsague X, Muñoz N, Iscovich J, Chopita N and Jmelnitsky A: Alcohol, tobacco, diet, mate drinking, and esophageal cancer in Argentina. Cancer Epidemiol Biomarkers Prev 3: 557-564, 1994

9. Wu M, Zhao JK, Hu XS, Wang PH, Qin Y, Lu YC, Yang J, Liu AM, Wu DL, Zhang ZF, Frans KJ and van't Veer $P$ : Association of smoking, alcohol drinking and dietary factors with esophageal cancer in high- and low-risk areas of Jiangsu Province, China. World J Gastroenterol 12: 1686-1693, 2006.
10. Sewram V, De Stefani E, Brennan P and Boffetta P: Mate consumption and the risk of squamous cell esophageal cancer in Uruguay. Cancer Epidemiol Biomarkers Prev 12: 508-513, 2003.

11. Castellsagué X, Muñoz N, De Stefani E, Victora CG, Castelletto R, Rolón PA and Quintana MJ: Independent and joint effects of tobacco smoking and alcohol drinking on the risk of esophageal cancer in men and women. Int J Cancer 82: 657-664, 1999.

12. Lee CH, Lee JM, Wu DC, Hsu HK, Kao EL, Huang HL, Wang TN, Huang MC and Wu MT: Independent and combined effects of alcohol intake, tobacco smoking and betel quid chewing on the risk of esophageal cancer in Taiwan. Int J Cancer 113: 475-482, 2005

13. Ganesh B, Talole SD and Dikshit R: Tobacco, alcohol and tea drinking as risk factors for esophageal cancer: a case-control study from Mumbai, India. Cancer Epidemiol 6: 431-434, 2009.

14. Cassetti T, La Rosa F, Rossi L, D'Alò D and Stracci F: Cancer incidence in men: a cluster analysis of spatial patterns. BMC Cancer 8: 344, 2008.

15. Barón AE, Franceschi S, Barra S, Talamini R and La Vecchia C: A comparison of the joint effects of alcohol and smoking on the risk of cancer across sites in the upper aerodigestive tract. Cancer Epidemiol Biomarkers Prev 2: 519-523, 1993.

16. Vioque J, Barber X, Bolumar F, Porta M, Santibáñez1 M, García de la Hera M and Moreno-Osset E for the PANESOES Study Group: Esophageal cancer risk by type of alcohol drinking and smoking: a case-control study in Spain. BMC Cancer 8: 221, 2008.

17. Pandeya N, Williams GM, Sadhegi S, Green AC, Webb PM and Whiteman DC: Associations of duration, intensity, and quantity of smoking with adenocarcinoma and squamous cell carcinoma of the esophagus. Am J Epidemiol 168: 105-114, 2008.

18. Wang Z, Tang L, Sun G, Tang Y, Xie Y, Wang S, Hu X, Gao W, Cox SB and Wang JS: Etiological study of esophageal squamous cell carcinoma in an endemic region: a population-based case control study in Huaian, China. BMC Cancer 6: 287, 2006.

19. De Stefani E, Muñoz N, Estève J, Vasallo A, Victora CG and Teuchmann S: Mate drinking, alcohol, tobacco, diet, and esophageal cancer in Uruguay. Cancer Res 50: 426-431, 1990.

20. Grønbaek M: The positive and negative health effects of alcoholand the public health implications. J Intern Med 265: 407-420, 2009.

21. Bruzzi P, Green SB, Byar DP, Brinton LA and Schairer C: Estimating the population attributable risk for multiple risk factors using case-control data. Am J Epidemiol 122: 904-914, 1985.

22. Engel LS, Chow WH, Vaughan TL, Gammon MD, Risch HA, Stanford JL, Schoenberg JB, Mayne ST, Dubrow R, Rotterdam H, West AB, Blaser M, Blot WJ, Gail MH and Fraumeni JF Jr: Population attributable risks of esophageal and gastric cancers. J Natl Cancer Inst 95: 1404-1413, 2003.

23. Lee CH, Wu DC, Lee JM, Wu IC, Goan YG, Kao EL, Huang HL, Chan TF, Chou SH, Chou YP, Lee CY, Chen PS, Ho CK, He J and Wu MT: Carcinogenetic impact of alcohol intake on squamous cell carcinoma risk of the oesophagus in relation to tobacco smoking. Eur J Cancer 43: 1188-1199, 2007.

24. Brown LM, Hoover R, Silverman D, Baris D, Hayes R, Swanson GM, Schoenberg J, Greenberg R, Liff J, Schwartz A, Dosemeci M, Pottern L and Fraumeni JF Jr: Excess incidence of squamous cell esophageal cancer among US Black men: role of social class and other risk factors. Am J Epidemiol 153: 114-122, 2001. 